IV.-Miscellaneous Observations on the Blood. By JoHn Davy, M.D., F.R.S., Lond. \& Ed., \&c.

(Read 6th March 1865.)

On a fluid of so much importance as the blood, observations with any pretension to accuracy can hardly be too often made and repeated, more especially when we consider its great instability, its little uniformity, and the differences of opinion entertained by physiologists respecting some of its most remarkable properties.

Such is the persuasion which has influenced me in engaging in the present inquiry, and in submitting its results to the Society.

I. On the Action of Water on the Red Corpuscles of the Blood.

As is well known, the red corpuscles are altered in form and appearance on admixture with water, the most obvious change being, that from discs they expand into globules.

In some trials made with the view to ascertain something more precise, I have selected the blood of birds, that chiefly of the common fowl and duck, the corpuscles of their blood, from their elliptical shape, being peculiarly fit, as it seemed, for the inquiry.

The first trials made were to ascertain the proportion of water that was required to effect any material change. The results obtained were the following :-

When one measure of water was added to one of serum holding red corpuscles in suspension, but few of them experienced an immediate change of form and became globular.

On the addition of two of water, the majority of the corpuscles underwent this change, a few only retaining their normal form.

On the addition of three of water, none of a normal form could any longer be seen; all that were visible were rounded, much reduced in apparent size, and were much less distinct; indeed, a nice adjustment was required to detect them. Many of them had a jagged outline; and from some there was a slight projection, suggestive of a rupture of their capsule.

Dried by evaporation at about $100^{\circ} \mathrm{Fahr}$. , very many of them were found to have recovered their original form and size. Some of them, however, appeared to be ruptured, the excluded nuclei adhering to their surface; others retained their nuclei, of irregular appearance; all appeared to be wasted. 
On the addition of four of water, the corpuscles were seen less distinctly, yet they were to be seen, the adjustment being as accurate as possible, and using a warm object-glass, a precaution needed to prevent the dimming of the glass ( $\frac{1}{8}$ th inch power) from the vapour rising from the fluid in such close proximity.

When more water was added, the only material difference that I am aware of was not in the effect on the corpuscles, but in their wider diffusion, thus increasing the difficulty of observing them. To counteract this, a portion of cruor was mixed with water in a tall vessel, stirred occasionally, and after having been some hours left at rest, the greater part of the coloured fluid-coloured by the solution of the colouring matter of the corpuscles-was drawn off. What remained afforded an interesting result. A drop of this fluid under the microscope exhibited much the same appearance as that from the addition of four parts of water ; and on drying at the same temperature, the appearances were also similar but more strongly marked, suggestive of ruptured capsules and the loss of their contents, with the exception, as in that instance, of some of them retaining their nuclei, these more or less altered. Most of the corpuscles, if that term be applicable to their remains, were circular or portions of circles, portions of them having been broken of. Some showed a rent, a few were elliptical, and with the exception of being wasted, but little altered in appearance.

The agency of water on the red corpuscles has commonly been attributed to imbibition or endosmosis, to solution of the soluble matter which these cells contain, and to exosmosis. The appearances which I have described seern to harmonise well with this view, with the addition of rupture of the cell-wall or capsule, and the occasional exclusion of the neuclei. They accord, too, tolerably with those noticed by Professor Lehmans, in his Physiological Chemistry, ${ }^{*}$ with the exception of two particulars. He states, that when largely diluted, the corpuscles become invisible under the microscope, which he attributes to their refractive power, after the action of water, differing but little from that of water itself. As already mentioned, when using certain precautions, $I$ have found them, only much less distinct. The other particular relates to their remains. According to him, these are mere shreds, and not empty and more or less broken capsules, as I have found them to be. The subject, it must be admitted, is one in the investigation of which it is not easy to obtain uniform and satisfactory results, there are so many interfering and disturbing circumstances concerned, not omitting the influence of the serum, and especially keeping in mind the powerful attraction the corpuscles have for water, and their hygroscopic properties; and further, the changes to which they are liable as dead matter, from the influences to which they are exposed.

As to the last mentioned, I have found that the longer the blood is kept, the

* Vol. ii. p. 184. 
smaller is the quantity of water that is required to alter their form. As to their hygroscopic property, this is shown by the simple experiment of breathing on them, or by exposing them over water for a few hours, keeping them, of course, out of contact with the water. In the instance of the warm vapour of the breath, one expiration is sufficient to deprive them, previously dried, of their elliptical form.

It is worthy of remark, that when the corpuscles are coloured by the addition of a weak solution of iodine, not only the action of the warm vapour of the breath is in a great degree arrested, but even the action of water, and this after immersion in water on a glass support for twelve hours, when they were found to retain their normal form, only slightly contracted, with their nuclei distinct. May it not be conjectured from this, that iodine medicinally used may operate in a degree similarly, and thus may arrest undue metamorphic disintegration?

II. On the Changes which take place in the Blood when excluded from the Air.

The changes to which the blood is subject when exposed to the air, at ordinary atmospheric temperatures, are pretty well known. To endeavour to ascertain what would happen were air as much as possible excluded, the following experiments were made :-

A bottle full of water, deprived of air by the air-pump, was emptied the instant before receiving blood from the divided cervical vessels of a barn-door fowl; so soon as full to overflowing, it was closed with a glass stopper lubricated with oil, and inverted in water.

During the first hour the blood retained its original hue, bright vermilion, and this throughout. After two hours the colour had lost something of its brightness. The following day the colour had become uniformly chocolate brown. The day after there was no appreciable change. The serum which had separated was of a wine yellow, and the crassamentum had contracted considerably. On the third day the serum was beginning to show a reddish tinge. From this day, viz., the 9 th of November, to the 4 th of December, the serum became of a darker red, and, like the crassamentum, was almost black, as seen by reflected light. During the time mentioned, the temperature of the room in which the blood was kept varied from about $55^{\circ}$ to $58^{\circ}$. The bottle was now taken out of the water, which was as clear as at first, showing that the closure was complete. The stopper was drawn out with ease, proving-as it was introduced when the blood was warm-that there was pressure from within rather than from without, though there was no appearance of any gas evolved. The serum decanted was of a dark purplish-red, as seen in thin layers by transmitted light, but black by the same light, and opaque, in a tube of one-half inch diameter. The crassamentum, of the same colour, was soft and easily broken up, and had, as well as the serum, an offensive putrid smell, but less so than if air had been allowed access 
With hydrate of lime, both yielded a strong ammoniacal odour. The blood corpuscles, as seen under the microscope, were found to have become rounded and globular. The fibrin seemed to be permanently dyed red; it retained this colour after having been well washed, and after maceration in water for many hours. Its structure was finely granular; it showed no appearance of fibres under gentle pressure, and viewed with a high power.

The experiment was repeated, using the blood of a turkey and also of a bullock. The results were similar. That on the blood of the turkey was of the same duration as the preceding. That on the bullock's blood was begun on the 14th of December, and ended on the 7th of January. In the latter instance, though no gas was evolved, the putrid blood, when subjected to the air-pump, entered into violent ebullition from the copious disengagement of air, and this even before the vacuum was nearly complete. It may also be mentioned, that a silver probe plunged into the clot became, after a few minutes, strongly discoloured, indicative thus of the presence of sulphuretted hydrogen.

A fourth experiment was made with the blood of a duck. In this instance, instead of emptying the bottle of water before receiving the blood, the water, deprived of air, was to a certain amount expelled by the blood as it flowed from the divided vessels. The specific gravity of the mixture was 1033. After having been kept from the $23 \mathrm{~d}$ of November to the 12th of January, at a temperature varying from about $40^{\circ}$ to $50^{\circ}$ and $55^{\circ}$, the changes observed on examination were so similar to those already specified that they need not be described.

A fifth experiment was made with the blood of a fowl. As in the last the blood was mixed with water; but it differed from the last in being subjected to the air-pump as soon as it had become sufficiently cool. No air was thus extricated. The bottle was again closed and inverted in water. This was on the 16th of February; it was examined on the 17th of March. Some difficulty was experienced in withdrawing the stopper. The blood bore marks of an incipient putrefaction; its smell was offensive, and some muriate of ammonia was formed on a plate of glass moistened with hydrochloric acid put over it during a few hours.

These results, all so well marked, seem to be nearly identical with those which occur when blood of the same temperature is exposed to the air, almost the only difference that I am aware of being in degree. The change of colour is the same, with the exception, that when exposed to the atmosphere, the blood at the surface, especially if it be venous, becomes florid before it darkens; the change of form of the corpuscles is the same, and the solution in the serum of their colouring matter. The same gases likewise are formed, and the same alkali is generated, accompanied by the characteristic putrid odour.

That blood should thus undergo change when air is excluded, is no more, perhaps, than might be expected when we reflect on its composition, and that oxygen 
is contained in it in a state, it is presumed, free to act and give rise to putrefactive fermentation.

What is more remarkable is the fact, that blood may be retained in the living body, stagnant, at rest, without undergoing similar changes, at a temperature so favourable to these changes. I may refer to Hewson's collected works, edited by Mr Gulliver, for instances of the kind. In a note, page 17, to mention one, the editor remarks, "occasionally blood is extravasated and stagnant in the living body for an indefinite time, and yet retains fluidity, as Mr Hunter and Mr CASAR HAwisiss have noticed." He adds, "I saw a case in a soldier, who had received a bruise in his loins, from his horse bolting with him over a bridge in Hyde Park; the injured part quickly swelled, evidently from effused fluid, which was let out twenty-eight days afterwards. It measured five ounces, was as liquid as blood just drawn from a vein, and coagulated in a cup in less than three minutes. The corpuscles were observed to be unchanged, and readily collected together in the usual way by their broad surfaces. Next day the clot was moderately firm, scarlet at the top, somewhat contracted, and surrounded by a little serum." What a contrast this presents to the blood from which atmospheric air was excluded in the experiments detailed! Cari the difference have been owing to the stagnant blood in the living body having been exposed to the action of the surrounding tissues, by which it is possible that, though a change may have been going on slowly in the blood, the degraded or altered particles may have been carried away as they were produced, leaving the residue in its normal state? I have witnessed something analogous when a mass of fibrin, enclosed in a muslin bag, has been immersed in water under a cock, from which there was a constant small stream keeping the water round the included fibrin in motion. During about a month that the fibrin was thus exposed at a temperature of about $40^{\circ}$, it had undergone little change; it was firm and only slightly tainted. In instances of aneurism, it is well known that not only the fibrin, but also the crassamentum enclosed in the sac resist for a long time putrid decomposition. May not this resistance be referred to the same cause? This explanation is submitted conjecturally. The fact that extravasated blood, from contusion and vascular rupture, is commonly absorbed with discoloration of the bruised part, may be adduced as somewhat in its favour. The physiologists of the School of IIunter would doubtless refer the liquidity of the blood, in the case in question, to the vitality of the blood; but that is a doctrine which at present is hardly tenable.

\section{On the Action of the Air-Pump on the Blood.}

The air-pump I have used in the trials I am about to describe is the same as that with which I made some former experiments on the blood, ${ }^{*}$ and, as then, it was in excellent order.

* Anatom. and Physiolog. Res. vol. ii. p. 214.

VOL. XXIV. PART I. 
The chief precautions taken were to receive the blood as it flowed from the divided vessels of the animal killed into phials, immediately after they had been emptied of water from which the air had been expelled by the action of the airpump, and after closing with a glass stopper, cooling the blood rapidly by immersion in water.

Though these precautions were taken, I believe they were not absolutely necessary for good results, as I find that when water exhausted of air is poured into a carefully washed phial from which water containing air has been poured out, on submitting it to the air-pump, no air is extricated either from the water or from the side of the phial.

The experiments on exhaustion have been made on the blood of the common fowl, of the duck, of the sheep, bullock, and pig; they have most of them been several times repeated.

The results have varied more than I could have expected, tending to show that the quantity of air extricable from the blood by the air-pump is far from constant, and depends on circumstances, some of which are appreciable, others obscure.

1. From the blood of the common fowl, the quantity of air disengaged has commonly been less than from that of the duck, sheep, bullock, and pig.

2. The blood of all the animals, when taken from them shortly after feeding, has commonly afforded more air than from animals of the like kind when fasting.

3. Florid blood, which it may be inferred is chiefly arterial, has yielded less air than dark blood, which probably is chiefly, venous, and, accordingly, that which flows first, when an animal has been blooded to death, less than that which flows last.

4. In a small number of instances, those of animals killed after a fast of many hours, the fresh blood yielded no air. In some of the trials which gave this result, the blood was mixed as it flowed with an equal quantity of water deprived of air.

5. In no instance have I witnessed the disengagement of air from fiesh serum, proving that the air, when extricated from the blood, is derived from the clot, and it may be presumed, from the red corpuscles which are entangled in it.

6. As might be expected, I have found the disengagement of air from the action of the pump more copious in summer than in winter; and also more copious from blood, the fibrin of which has been broken up by having been agitated with shot previously freed from adhering air, than from the clot left entire. In the instance of the blood of the common fowl, which coagulates rapidly, affording a firm coagulum, even the puncturing of it makes a difference; air then escapes, which before was retained.

7. In many instances, blood which had yielded air on exhaustion, has, after exposure for a few hours to the atmosphere, on repetition of the exhaustion, 
ceased to yield air, and this when the first trial was stopped before the exhaustion of the air was nearly complete. This result, seemingly paradoxical, may have been owing to ammonia formed, which may have fixed carbonic acid; and that ammonia was formed, was proved by the hydrochloric test and the production of muriate of ammonia. It has been witnessed in the instance of both venous and arterial blood, but most remarkably in the latter, and in warm weather oftener than in cold. In support of the explanation offered, I may mention an experiment on the blood of a calf, which had no food for about twenty hours before it was killed. This blood,-it was arterial, - even at first gave off no air on careful exhaustion. It was kept under an exhausted receiver from the $23 \mathrm{~d}$ of April to the 13th of May, during the whole of which time it gave off no air, though the vacuum was as perfect as it could be made, and the pump was worked daily. At the end of this time the serum had become dark red, and on examination the blood was found in a state of incipient putrefaction and giving off ammonia.

What struck me as most remarkable in these experiments with the air-pump, was the comparatively small quantity of air, in most instances, disengaged from the blood, and its total absence in others, taking into account the quantity of carbonic acid liberated in the lungs during life in normal respiration, and also the quantity of air, both oxygen and carbonic acid, found in the blood by the German physiologists. Difference of temperature, comparing that of the hot blood circulating in the lungs in birds as high as $106^{\circ}-108^{\circ}$, and in the sheep, ox, and pig, as high as $104^{\circ}-106^{\circ}$, with that of the blood of the same animals cooled to $50^{\circ}$ $55^{\circ}$, may partly account for the result first referred to, but the second adverted to I cannot attempt to explain.

Besides the foregoing trials with the air-pump, I have made some on the bloodcorpuscles, using very small quantities suspended in serum on a glass support. The corpuscles were from the blood of the animals already mentioned, and also of the frog and common trout. The results were all nearly similar: so long as the corpuscles were floating in serum there was no appreciable change of form, but if they were kept some hours under the exhausted receiver until they were left apparently dry on the object-glass, then a change was perceptible in them. Under the microscope they were found to have become greatly reduced in size, so as to be seen with difficulty, and not without the nicest adjustment, and also altered in form-the elliptical, as those of the bird, the fish, and batrachian, having become rounded. These changes were very similar to those produced by the action of water, and they may be accounted for, perhaps, on the idea that they were owing to the hygroscopic quality of the corpuscles. I may further remark that the effects on the corpuscles of the blood of the several animats tried some-

* I have found the temperature of the blood of a pig, flowing in a full stream, $106^{\circ}$. The pig was in high condition; the blood used was from it. 
what varied; it.seemed greatest in those of the common fowl, least in those of the ox.

\section{On the Effect of a Low Temperature on the Blood.}

It was ascertained by Hewsor that the blood, by rapid freezing, is not deprived of its property of coagulating when thawed; $*$ besides this and the change of form of the corpuscles from refrigeration which $I$ have observed, $f$ I am not aware that any thing has hitherto been published respecting the agency of a low temperature on this fluid.

During the frost which prevailed in the Lake District the winter before last, from the $2 \mathrm{~d}$ to the 10 th of January, I had an opportunity of renewing the inquiry. The blood used was that of the turkey, of the common fowl, and of the sheep. The most remarkable result obtained was that a low temperature, like a high temperature, appears to promote not only a change of form of the red corpuscles, but also a change of composition, as indicated by the production of ammonia and the solution in the serum of the colouring matter of the blood. As the changes were the same whichever blood was the subject of experiment, I shall restrict myself to what was observed in the trials on that of the common fowl. On the 4th of January a wine-glass was nearly filled with the blood of a full-grown fowl as it flowed from the divided great cervical vessels; it coagulated in less than two minutes. $\Lambda$ plate of glass, moistened with a drop of dilute hydrochloric acid, was placed over it. After ten minutes there was a copious deposition of dew on its inner surface, vapour from the warm blood beneath, and there condensed. On examination with microscope, after evaporation, not a trace of muriate of ammonia could be detected. The trial was repeated, and for six hours in the open air, at the temperature of $28^{\circ} \mathrm{Fahr}$; now a trace barely of the salt was found. The blood was moderately florid, preserving its original appcarance, and was not yet frozen. It was left out during the night. The temperature during the time, as shown by a register thermometer, was as low as $12^{\circ}$; on the following morning, at 9 A.M., it had risen to $18^{\circ}$. The blood was found to be frozen hard and thoroughly; it was greatly darkened in colour, and had lost entirely its florid hue. Distinct crystals of muriate of ammonia, and these not a few, were detected on the covering glass after the evaporation of the acid, and the red corpuscles from elliptical had become circular and globular.

The observations wcre continued until the morning of the 10th, when a thaw set in. The blood was examined twice daily, viz., at 9 A.M. and at 3 P.M. During the period the temperature was always below $20^{\circ}$, but not lower than $15^{\circ}$, excepting once, as already mentioned. The day temperature ranged as high as $27^{\circ}$, it was never lower than $22^{\circ}$. At the former temperature, a softening of the blood was

* Hewson's Works, p. 25.

$\dagger$ Physiological Researches, p. 369 . 
observed from incipient thaw. During the whole time, as denoted by the test employed, ammonia was evolved, and, as well as I could judge, the lower the temperature the larger was the quantity. I need hardly remark that there was no appreciable contraction of the crassamentum, no further separation of serum after congelation had taken place. The serum which first exuded before congelation-a very small quantity - after having been frozen, became coloured, and, finally, of a red nearly as dark as the general mass, and this owing in part to blood corpuscles suspended in it of altered form, and in part to solution of their colouring matter. After thawing, the blood had no smell indicating putridity, nor did it discolour silver; yet it continued, at a temperature of $50^{\circ}$, to evolve ammonia, and much in the same proportion as when frozen. Now, however, the contraction of the crassamentum, i.e., of its fibrin, before arrested, took place, and to an extent seemingly differing but little from what would have occurred had the blood not been frozen. The blood corpuscles now were so reduced in size, and had become so transparent, that unless dried, they were seen with difficulty, and not without the most accurate adjustment.

'lhese results, viz., the disengagement of ammonia, and, we must infer, its formation, when blood is frozen, are hardly such as could be expected; and they are the more remarkable, as seeming to be independent of putrefaction and the action of oxygen, and owing to a new arrangement of elementary parts produced by a low temperature, one ranging from about $50^{\circ}$ to many degrees below the freezing point. That congelation was not essential to the formation of the ammonia was shown in other experiments, in which, when blood was exposed to a temperature ranging in one trial from $32^{\circ}$ to $34^{\circ}$, the volatile alkali was produced, and in others at a temperature varying from $40^{\circ}$ to $50^{\circ}$; and, in the latter, even when continued several days, without any indications of putridity, judging from the absence of the smell such as denotes putrefaction, and from silver immersed remaining untarnished.

At first view what has been described may seem anomalous, yet the results are not without analogies. The potato, as is well known, becomes sweet from the conversion of starch into sugar by "frosting ;" and the ripening of the grape, the sweetening of its juice, it is also well known, is hastened by the setting in of frost at the time of the vintage in Switzerland, and in other countries with a similar climate. The formation of peat is another example of the efficiency of a comparatively low temperature in producing new compounds. Familiar with the effects of heat-i.e., of a high temperature-as an active agent, it is not perhaps surprising that cold-i.e., a low temperature-should be little thought of except as the opposite and the antagonist of heat, disregarding the fact that they differ merely in degree; and how inconsiderable that is, whether measured by our sensations or by the thermometer.

After witnessing the effects of congelation on blood, the question occurred, Is VOL. XXIV. PART I. 
meat liable like it to change from freezing - that change which the evolution of ammonia indicates? As it is well known that meat may be kept for weeks frozen without being spoiled as an article of diet, the obvious answer was in the negative. The only experiments I have made have afforded results leading to the same conclusion. A portion of fresh mutton, cut into small pieces, was exposed on the 6th of January to the open air. During the following night the register thermometer was as low as $16^{\circ}$; the next morning it was $22^{\circ}$. The meat was not frozen; its fibre was soft and flexible; a bare trace of muriate of ammonia was found on the glass above it prepared as a test of the volatile alkali. Before night it became frozen and rigid, and it continued so until the thaw began on the 10th. Examined twice daily, no traces could be detected of the production of ammonia. The fibre, indeed, was evidently softened, and its striated structure, as seen under the microscope, was less conspicuous, so much so, that without a good light and a careful adjustment it could not be seen.

A like question occurred respecting manures-Does frost arrest their decomposition? I have made trial of stable-dung, and have found it when frozen to exhale ammonia in an unmistakable manner, proving that a low temperature, as in the instance of blood, promotes its decomposition, or that change on which the evolution of ammonia depends. A similar result has been obtained from the exposure of impure lithate of ammonia (the urinary excrements of the pelican), of the mixed excrements, partly urinary, partly alvine, of the barn-door fowl, and of guano. From all of them, using the same test, the production of ammonia was conspicuous. Should not these results suggest the propriety of reconsidering the treatment of manures, and if not the time of their application to the land, at least whether an addition should not be made to them to fix the ammonia?

\section{On the Action of Ammonia on the Blood.}

Since the hypothesis has been advanced, that the escape of ammonia from the blood is the cause of its coagulation, additional interest is attached to the action of the volatile alkali on this fluid.

The following experiments have been made with a view not so much to test the correctness of that hypothesis, as to show what are the effects of ammonia on the blood as a whole, and on its several parts :-

1. On the Entire Blood.-On the 8th of December $241 \mathrm{grs}$. of the blood of a duck were received, as it flowed from the divided cervical vessels, into a bottle containing $72.5 \mathrm{grs}$. of aqua ammoniæ of sp. gr. $\cdot 95$. The bottle was immediately closed with a glass stopper. This was at 10.37 A.M. At 12.15 P.M. a semi-fluid viscid coagulum had formed, of a rich Turkey-red colour. A glass rod applied to it, it yielded to gentle pressure, without adhering to, or in the slightest degree soiling the rod. At 2.30 P.M. it was somewhat firmer. At 10 P.M. it was more 
so ; now, when the bottle was turned on its side, it ceased to flow. On the following day it was so firm that it bore inversion without flowing; no serum had separated. Examined on the 13th of December, the only change perceptible was that it was rather firmer. On the 1st of January it was more carefully examined. On withdrawing the stopper, as might have been expected, the smell of the ammonia was very powerful, indeed unendurable. The coagulum was found of the consistence of a pretty firm jelly, readily yielding to pressure, but not adbering to the glass rod impressing it. The whole mass was easily removed, retaining its form unbroken; and such was the adhesiveness of its substancei.e., of its particles to one another-that the mass admitted of being divided with a scissors without its soiling the instrument. A portion of it put into water did not immediately colour the water; from black it became dull brown. Examined with the microscope under compression, it exhibited a finely granular surface, through which were scattered globules of a less diameter than the blood corpuscles -these, it may be inferred, contracted.

In other experiments, made within a few days of each other, with smaller proportions of the volatile alkali, the effect has been found to vary.

When 12 grs. of aqua ammonix, of the same strength as that last mentioned, were mixed with 465 grs. of the blood of a turkey, the instant it was shed, the coagulation was retarded about 20 minutes. On withdrawing the stopper the following morning there was a strong smell of ammonia; the crassamentum was found of tolerable consistence, and was surrounded and covered with red serum, which owed its colour chiefly to red corpuscles suspended in it of a globular form, a change from their normal form evidently owing to the action of the alkali. Examined again after twenty days the coagulum was found firmer ; it admitted of being taken out as an entire mass.

In a third experiment 2.5 grs. of aqua ammoniæ were mixed, as in the former instances, with 572 grs. of the blood of a fowl. After two hours the blood was found feebly coagulated and viscid, in a semifluid state, sluggishly flowing like tar. When a portion of it was poured into water, it did not mix with the water, but kept entire, retaining its viscidity. What remained, examined the following morning, was found divided into a somewhat denser crassamentum, still semifluid and viscid, and a red somewhat viscid serum, abounding in red corpuscles, more or less altered in form, many of them diminished in volume, and almost all of them rounded. Both the soft coagulum and the serum smelt of ammonia.

A fourth experiment was made on the blood of a sheep, after the same manner as the preceding. The quantity of the aqua ammoniæ was 1 gr., of the blood 587 grs. Examined after about half-an-hour, the blood, still warm, was found pretty firmly coagulated, and already some serum had separated. The glass stopper was withdrawn, and instantly after a plate of glass, moistened with dilute hydrochloric acid, was placed over the mouth of the bottle, and left for two minutes. 
Now, after evaporation, crystals of muriate of ammonia, of a large size and in abundance, were found on it.

A fifth experiment, similar in manner to the last, with the exception that the aqua ammoniæ (1 gr.) was spread as much as possible over the inside of the phial, was made on the blood of a fowl (590 grs.) The blood coagulated in eight minutes, and pretty firmly. Another portion, caught in a wine-glass, coagulated in about a minute. Each was tested for ammonia, as in the preceding trial. The blood in the wine-glass, after five minutes-the time that the acid was kept over it-afforded no distinct trace of ammonia. The blood in the bottle, after one minute, afforded ample proof of the evolution of ammonia in the large crystals of the muriate which were formed on evaporation on the incumbent glass. The contrast, indeed, was very striking, comparing the blood with and without the addition of ammonia, as thus tested, and also by test-paper; the one, the former, having no effect during a minute that moistened test-paper was held over it; the other, in the same time, producing a decided alkaline reaction. On the following morning the crassamentum in the bottle was found slightly contracted, though less than that in the wine-glass; some reddish serum had separated ; the blood corpuscles, whether suspended in the serum or retained in the clot, were little if at all altered.

From these experiments it would appear that the effect of aqua ammoniæ varies as to the quantity used, and this in a manner that could hardly be expected; 31 per cent. occasioning a thick adhesive coagulum, with a change of form of the red corpuscles, without the separation of any serum; 2.5 per cent. retarding the coagulation many minutes, but not preventing the separation of serum and a certain contraction of the crassamentum; $0 \cdot 44$ per cent. retarding the coagulation and rendering the coagulum soft and viscid, barely semifluid, with little separation of serum, and that viscid; lastly, $0 \cdot 17$ per cent. had little effect, except that of retarding for a few minutes the coagulation,- - the coagulum, when formed, having very much its normal appearance.

2. On the Fibrin of the Blood.-The fibrin used was obtained by washing the clot which had formed in the first experiment. In its moist state it was slightly viscid. By drying it lost 93 per cent. ; $2 \cdot 5$ grs. thus dried were put into a phial with 273 grs. of aqua ammoniæ of sp. grav. 89 , and secured by a glass stopper. After eleven days it was not apparently diminished in volume : $41 \mathrm{grs}$. of the clear fluid decanted and evaporated yielded only $1 \mathrm{gr}$. As the fluid became concentrated during the process, which was conducted at a low temperature, its fluidity diminished, and when reduced to a drop it was still transparent. In its dry state it appeared as a transparent film, and as seen under the microscope with a high power it had a finely granular appearance.

A second experiment was made on the same fibrin in its moist state, using in place of aqua ammoniæ alone a dilute solution, consisting of $65.4 \mathrm{grs}$. of the alkali, 
and of $30 \tilde{z}$ grs. of water. The fibrin was equal to 31 grs. After ten days its volume seemed little diminished-58.4 grs. of the clear fluid evaporated left $\cdot 1 \mathrm{gr}$. The undissolved residuary portion, constituting so large a proportion of the whole, was soft, glutinous, and adhesive; it might be called ropy, as it allowed of being drawn out, and when agitated by a circular motion, it rose spirally in the liquid. It thus differed from the dried fibrin, which was softened in a slight degree, but not rendered glutinous. Examined after thirty-four days, it seemed little altered in bulk, and nowise in its properties. The fluid was slightly viscid : a portion of it, $41.4 \mathrm{grs}$, evaporated to dryness, yielded only $05 \mathrm{gr}$. It became slightly turbid during evaporation at a temperature of about $180^{\circ}$, and when the ammonia was driven off, it lost the little viscidity it before had. The smaller proportion of residue in this instance might have been owing to the circumstance that the phial holding the fibrin and the dilute aqua ammonix not being firmly corked, some of the ammonia might have escaped.

These results demonstrate how feeble is the solvent power of ammonia on fibrin. Many other experiments which I have made, of which an account is hardly needed, have been amply confirmatory of the fact, and also of the wellknown effect of ammonia in rendering fibrin viscid and glutinous, and of increasing its transparency. This last effect should be kept in mind, otherwise, as the refractive power of fibrin differs but little from that of water, it may in some instances be imagined to be dissolved, when it is only diffused.*

3. On the Serum of the Blood.-On this fluid the effect of ammonia is less distinct. It appears to diminish rather than to increase the viscidity of the serum, as is shown by the following experiment: a portion of the serum of the blood of a pig, equal to 314 grs., was mixed with $274 \cdot 4$ grs. of aqua ammonie of sp. gr. 89 , in a glass-stoppered phial ; and about an equal quantity of the serum of the same blood was poured into a similar phial. This was on the 30th March. Each was shaken daily: froth was produced in each instance, but that from the ammoniacal mixture subsided more rapidly than that from the serum alone; and the longer the trial was continued-it was continued more than a month-the more marked was the difference.

At the end of this time the ammoniacal mixture had deposited a white

* When well-washed fibrin, still slightly coloured by the colouring matter of the blood, is placed under the microscope, it appears to consist of translucent granules forming under gentle pressure a connected tissue. On the addition of aqua ammoniæ it becomes clear and transparent, like jelly, with a brightening of its colour. Compressed, it shows elasticity, and when extended by continued pressure, so as to be very thin, its appearance is hyoloid; no granules are to be seen in it except a few scattered ones, which, it may be, were derived from blood corpuseles.

Fibrin which has been rendered viscid by ammonia, after the removal of the ammonia by repeated washing, gradually contracts, and from being transparent becomes, in consequence of condensation, opaque, or nearly so. Thus contracted it often exhibits an imitative form, like that of hydatids. Its retention of the colouring matter of the blood is remarkable; it is greater even than that of the capsule or walls of the corpuscles.

VOL. XXIY. PART I. 
matter, which was readily diffused on gentle agitation, rendering the fluid, which was before transparent, turbid.

Under the microscope the deposit exhibited thin crystalline plates, their length exceeding their width about a third, some minute spicula, somewhat like raphides, and some granules. As the matter was not viscid, it may be inferred that fibrin did not form a part of it.

The transparent fluid separated by decantation from this sediment yiclded a coagulum with the sulphuric, muriatic, nitric, and acetic acids, added each in slight excess, that is, in a quantity a little more than was sufficient to neutralise the ammonia. The precipitate was redissolved by the sulphuric and muriatic acids, and in great part by the nitric-these acids concentrated-but not by the acetic.

When the clear ammoniacal fluid was boiled until the whole of the volatile alkali was expelled, it was rendered gelatinous, that is, the coagulum formed was soft and transparent, like the albumen of the eggs of some birds similarly treated.

The same fluid, evaporated at a low temperature, left a brownish transparent matter, which was soluble almost entirely in water. The solution frothed when boiled and gelatinised. It had a slight alkaline reaction, like serum, and had no unpleasant smell. Evaporated again, little of it was redissolved on the addition of water, and still less on repeating the operation-thus resembling ordinary serum.*

The serum without the addition of ammonia, kept during the same time, had also yielded a deposit, which was of a greyish hue, and under the microscope exhibited only amorphous particles. The fluid had acquired a reddish hue, and had an offensive putrid smell, and it afforded when boiled a firm coagulum.

Comparing, then, the two, it appears that ammonia renders serum less viscid, prevents its putrefaction, $\uparrow$ and modifies in some degree its coagulable property. Whether the serum of the blood of other animals under the influence of ammonia would show the same properties, I have not ascertained with sufficient accuracy. From the few comparative trials I have made, I am disposed to infer that there would be no material difference.

4. On the Red Corpuscles of the Blood.-On these the effect of the volatile alkali is more decided, as is shown by the following experiment,--one of the

* Serum of blood, such as $I$ have tried, and $I$ have made many trials, on first evaporation affords a residue which is almost entirely soluble in water, but on repetition again and again, it is so altered as to become insoluble.

† Ammonia does not appear to arrest entirely the putrefactive decomposition of the blood: thus a mixture of 257 grs. of blood, and of $2 \cdot 5$ grs. of aqua ammonia, the subject of the third experiment, after having been kept twenty days, had, besides an ammoniacal odour, an offensive smell, indicative of incipient putrefaction. In great excess, it certainly retards the change in the instance of the entire blood. and in a great degree in the instance of the fibrin, and in that of the serum. A portion of the coagulum left from the first expcriment and kept three months, had, at ter the ammonia had been rapidly expelled, an offensive odour, only in a slight degree. 
many which I have made. The cruor used was from fresh bullock's blood, its fibrin separated in the usual way; 45 grs. of it were mixed with 48 grs. of aqua ammoniæ. The colour was immediately darkened, so much so, that by reflected light it appeared almost black; by transmitted, of a garnet-red, similar to the change of colour observed when the entire blood was used, and it was accompanied by the same alteration in the corpuscles, these being reduced in size and rendered globular. Another obvious effect was an increase of viscidity. Examined after eight days, and again after twenty-four, the only further change noticeable was the disappearance of the corpuscles, as if they had in great part been dissolved ; they were not to be seen under the microscope; minute granules only were visible, and these were seen only after evaporation.

It is worthy of remark, that when the whole of the volatile alkali was expelled by heat at a temperature below $160^{\circ}$, and the residuary fluid was tried by test-paper, only the feeblest alkaline reaction was observable; in this respect, differing from the serum, which under the same circumstances showed a distinct alkaline reaction. The solution was coagulated at a temperature of about $160^{\circ}$. Another portion evaporated at a low temperature was resoluble, $i . e .$, the colouring matter; seeming to show that this matter had suffered no change from the action of the volatile alkali.

The bearings of the results of these several experiments on the hypothesis adverted to, hardly need be dwelt on, they are so obvious. Seeing that ammonia, in so large a quantity as that used in the first experiment, did not prevent the coagulation of the blood, or, in other words, of its fibrin-its coagulable part-it would be strange, indeed, if the escape of a very minute quantity of the volatile alkali, hardly an appreciable one at most, should be the cause of the phenomenon.

Considering that ammonia renders the fibrin viscid and alters the shape of the red corpuscles, is there not ground for caution as regards its medicinal use, and of more than doubt of its efficacy when administered with the intention of dissolving a coagulum in cases of thrombosis? The marked difference as to alkaline reaction of the serum and cruor, as already mentioned, was suggestive of analogy between the blood and the contents of the egg. It is stated that an aqueous solution of the colouring matter of the former is neutral.* Whatever care I have taken in preparing it, draining off the serum as much as possible from the clot before the action of water, I have always found it feebly alkaline. $f$ Nor is this surprising, considering the impossibility of getting rid of all the serum by drain-

* Brande and Taylor's "Chemistry," 1863, p. 833 .

$\uparrow$ In one experiment, the clot, from six ounces of bullock's blood, after draining off as much as possible of the serum, was cut into small pieces and macerated in water, using the ordinary means to separate the fibrin. The solution formed, loaded with colouring matter, was evaporated at a temperature below $160^{\circ}$, until reduced to the sp. gr. 1033 ; its alkaline reaction then was very slight, so as to be hardly discernible when the delicate test-paper used was dried.

After evaporation of the solution to dryness, the residue was exposed to the fire in a platina capsule. In its charred state, after it had ceased to burn with flame, its particles were slightly 
ing. Further, I have found the ash of hæmatine prepared by a more elaborate process also feebly alkaline; and the latest analyses of the several ingredients of the blood, those most to be depended on, indicate the same.* May not this difference, slight though it appears, warrant the conjecture, that as in the egg, so in the blood, there may be an action of a galvanic kind between its several proximate parts? And may not the differences which are known to exist between the serum and the red corpuscles be adduced in favour of the conjecture?

\section{On the Coagulation of the Blood.}

Of the many hypotheses which have been advanced at different times to account for the coagulation of the blood, each has been supported, as hypotheses usually are, by some facts, but few of them have for any length of time maintained their ground, facts having been adduced hostile to them.

Of the latest hypotheses brought forward, one is that of Dr Richardson, briefly designated the ammonia-theory, to which I have already adverted; another is that of Professor LISTER, in which he considers the phenomenon as mainly depending, out of the body, on a kind of catalytic action produced by the contact of any foreign substance, and, within the body, as owing to an analogous cause, contact with a part, either dead or quasi dead,-as he supposes a tissue to be under the influence of inflammation. $t$

This hypothesis, as it appears to me, is open to certain objections. I shall now notice merely a few of the facts which seem to me most opposed to it.

1. Were it true, ought not the phenomenon of coagulation to take place in every instance in which dead matter comes in contact in the living body with the blood? Instances of ossification, in which concretions of phosphate of lime are formed in the arterial coats, and often project into the vessels themselves,-concretions differing but little from the "tartar," deposited so often on the teeth, and inorganic,-are familiar to every one acquainted with pathological anatomy, and yet in the majority of these cases the coagulation of the blood has not taken place

2 . In instances of aneurism, with a rupture of the vessel, the seat of it, a coagulum of blood is invariably formed, though in contact with parts which, it may be presumed, until the contrary is proved, still retain their vitality.

3. Examples of the coagulation of the blood in the veins, in the arteries, and in the ventricles of the heart, during life, in persons reduced to a feeble state by

attracted by the magnet, the coal after cooling having been reduced to powder. The particles of its ash, after the charcoal had been burnt off, were also similarly attracted. The magnet used, it may be mentioned, was a needle that had been magnetised by a foetal torpedo, and which (as the result showed) still retained its power, after the elapse of 32 years. The residuary ash, on the addition of a little water, showed so feeble an alkaline reaction, that it was hardly as well marked as that of the saliva.

* Lehurann's “Physiological Chemistry,” ii. pp. 160, 212.

$\dagger$ Proceedings Roy. Soc. Vol. xii. p. 580. 
disease, are not of unfrequent occurrence, and this often without any apparent lesion in the coats of the vessels themselves, or in the lining membrane of the ventricles.

4. Confirmatory of the last, many examples are on record of the blood, in its coagulated state after death, having been found broken up in the left ventricle of the heart, proving that its coagulation must have taken place whilst the heart was still forcibly acting, and this in cases in which the organ appeared to be sound.*

5. Certain poisons influence the coagulation, some accelerating it, some retarding it. As an example of both, may be mentioned the poison of a snake, the tic-polonga of Ceylon (Daboia Russellii, Gray), which on fowls acts with extreme rapidity, so much so, that simultaneously with their death, it occasions the coagulation of the blood in the heart and great vessels, and this even before the former has ceased to act; whilst, in larger animals, such as the dog, in which it takes effect less rapidly, causing death in an hour instead of about a minute, it has a contrary influence, that of preventing the coagulation of the blood. $\uparrow$ There are other considerations which seem to me to cast a doubt on the accuracy of this hypothesis. To reconcile it with certain facts, its author is under the necessity of assuming that a clot is a "living tissue in relation to the blood ;" if so, then does it not follow, in strictness of reasoning, that such must be its state under all conditions, whether formed within the body during life, or in blood abstracted by the ordinary operation of blood-letting; and he is further under the necessity of assuming that inflamed parts are quasi dead parts, or, in other words,-and they are his-" have lost for a time their vital properties, and comport themselves like ordinary solids."

The vagueness, moreover, of the hypothesis renders it open to objection. The referring the phenomenon to a catalytic action, seems to be little more than the accounting for what is obscure by that which is equally or hardly less obscure.

To conclude, I fear it must be confessed that, strictly speaking, the theory of the coagulation of the blood, its vera causa, is still an unsolved problem, there being, to all the hypotheses which have hitherto been propounded, opposing facts logically in strictness prohibiting the establishment of any one of them.

* See the author's Anatom. and Physiolog. Research, ii. $196 . \quad+$ Idem, vol. i. p. 123. 